\title{
Very Late Antigen-4 in CD18-Independent Neutrophil Emigration during Acute Bacterial Pneumonia in Mice
}

\author{
Sadatomo Tasaka, Sarah E. Richer, Joseph P. Mizgerd, and Claire M. Doerschuk
}

Division of Integrative Biology, Department of Pediatrics, Rainbow Babies and Children's Hospital and Case Western Reserve University, Cleveland, Ohio; and Physiology Program, Harvard School of Public Health, Boston, Massachusetts

\begin{abstract}
This study tested the hypothesis that very late antigen (VLA)-4 mediates CD18-independent neutrophil emigration into the airspaces induced by either Streptococcus pneumoniae, a stimulus that induces primarily CD18-independent neutrophil emigration, or Escherichia coli, toward which only $20-30 \%$ of the total number of neutrophils emigrate through CD18-independent pathways. In wildtype (WT) mice, VLA-4 expression was less on neutrophils that emigrated into the airspaces than on circulating neutrophils. Vascular cell adhesion molecule-1 (VCAM-1) mRNA, the major endothelial cell ligand for VLA-4, increased more in E. coli than in S. pneumoniae pneumonia. VCAM-1 protein expression was not detected in capillaries, the major site of neutrophil emigration. Neutrophil emigration during $E$. coli or $S$. pneumoniae pneumonia was similar in mice given antibodies against both CD18 and VLA-4 compared with mice given the anti-CD18 antibody and a control antibody. However, in hematopoietically reconstituted mice with both WT and CD18-deficient neutrophils in their blood, the migration of CD18-deficient neutrophils in response to S. pneumoniae was slightly but significantly less in animals pretreated with the anti-VLA-4 antibody than in those receiving a control antibody. These data suggest that VLA-4 plays a small role in CD18-independent neutrophil emigration, but the majority of CD18-independent neutrophil emigration induced by bacteria in the lungs occurs through VLA-4independent mechanisms.
\end{abstract}

Keywords: inflammation; phagocytes; bacterial infection; leukocyte adhesion molecules; mice

Neutrophil emigration out of the bloodstream into sites of inflammation is governed by adhesive events involving receptorligand pairs of adhesion molecules on neutrophils and endothelial cells $(1,2)$. The integrins are a family of cell surface molecules that mediate cell-cell and cell-extracellular matrix adhesion $(3,4)$. Each integrin consists of an $\alpha$ and a $\beta$ heterodimer that bind to one or more ligands. The leukocyte $(\beta 2)$ integrins are heterodimeric transmembrane glycoproteins consisting of one invariant $\beta 2$ (CD18) chain and one of four possible CD11 chains (CD11a, -b, -c, or $-d$ ). The $\beta 2$ integrins are required for acute neutrophil emigration under most circumstances $(1,5,6)$. In the lungs, however, neutrophil emigration occurs not only via CD11/CD18-dependent, but also through CD11/CD18-independent, pathways $(6,7)$. The stimulus appears to determine which adhesion pathway will be selected. Neutrophil emigration in response to Escherichia coli, E. coli lipopolysaccharide (LPS), Pseudomonas aeruginosa, phorbol ester, IgG immune

(Received in original form May 9, 2001; accepted in final form April 15, 2002)

This work was supported by NHLBI HL 48160, HL52466, and a Clinical Scientist Award (C. M .D.) in Translational Research from the Burroughs Wellcome Fund. $S$. T. is the recipient of a fellowship from the Sasakawa Health Science Foundation.

Correspondence and requests for reprints should be addressed to Claire M. Doerschuk, M.D., Rainbow Babies and Children's Hospital, Room 787 (MC 6003), 11100 Euclid Avenue, Cleveland, $\mathrm{OH}$ 44106. E-mail: cmd22@po.cwru.edu

Am J Respir Crit Care Med Vol 166. pp 53-60, 2002

DOI: $10.1164 /$ rccm.2105034

Internet address: www.atsjournals.org complexes, or interleukin-1 (IL-1) is inhibited by $60-80 \%$ when the function of CD11/CD18 is blocked, implying that $20-40 \%$ of neutrophils migrate through CD18-independent pathways (6). CD18-independent pathways are even more critical in neutrophil emigration induced by Streptococcus pneumoniae, Staphylococcus aureus, hydrochloric acid, hyperoxia, or C5a complement fragments, where CD18 blockade does not alter neutrophil emigration at all $(6,7)$. The adhesion molecules that mediate the CD18-independent pathways have not been identified.

The integrin family also contains the $\beta_{1}$ integrins, each of which contains a $\beta_{1}$ subunit associated with an $\alpha$ subunit. The integrin $\alpha_{4} \beta_{1}$ (very late antigen-4 [VLA-4]; CD49d/CD29) is a member of this subfamily and is expressed at substantial levels on mononuclear leukocytes (8-10). Its primary endothelial cell ligand is vascular cell adhesion molecule-1 (VCAM-1), and it also recognizes fibronectin (11). VLA-4 has important roles in monocyte and lymphocyte rolling and adhesion to endothelium. In the lungs, VLA-4 is required for eosinophil and T cell recruitment in the late-phase response and in airway hyperresponsiveness induced by antigen sensitization and challenge $(8,12-14)$.

Recent reports have described the expression of VLA-4 on neutrophils and a role for VLA-4 in neutrophil emigration during inflammation, both in vitro and in vivo. Kubes and colleagues demonstrate that VLA-4 is upregulated during the process of transendothelial cell migration in response to formyl-methionyl-leucyl-phenylalanine (fMLP) and anti-VLA-4 antibodies partially block the CD18-independent adhesion of these emigrated neutrophils (15). Neutrophils induced to express VLA-4 by treatment with dihydrocytochalasin B and fMLP adhered to tumor necrosis factor- $\alpha$ - (TNF- $\alpha)$-treated endothelial cells through a mechanism requiring VLA-4 and CD18 (16). In vivo, blockade of VLA-4 by specific antibodies completely inhibits neutrophil emigration induced by subcutaneous zymosan-activated serum, TNF- $\alpha$, or LPS (17). Blockade of VLA-4 attenuates the fraction of neutrophil emigration during adjuvant-induced arthritis that is not inhibitable by anti-CD18 antibodies, suggesting that VLA-4 can mediate CD18-independent neutrophil emigration (17-19).

Very recently, Ridger and colleagues (20) demonstrated that blockade of the $\beta 1$ integrins (CD29) prevented neutrophil emigration in response to either LPS (which evokes primarily CD18-dependent emigration) or $\mathrm{KC}$, a chemokine that induces CD18-independent emigration. Elegant electron microscopic studies revealed that $\beta 1$ integrins were involved in the migration of neutrophils from the interstitium to the alveolus rather than in neutrophil-endothelial cell adhesion. Further studies demonstrated that different CD49 ( $\alpha$ integrin) components were used in response to LPS and KC; CD49b and CD49d ( $\alpha 4$, VLA-4) were important only in KC-induced neutrophil emigration, whereas CD49e and CD49f inhibited responses to both KC and LPS (20). Studies by Burns and colleagues (21) also supported a role for VLA-4 as well as for $\alpha 5 \beta 1$. Their studies used an unusual model of LPS pneumonia 
in rats where approximately $80 \%$ of the migration still occurs despite blockade of CD18, and over half of the CD18-independent accumulation of neutrophils within the bronchoalveolar lavage (BAL) fluid was prevented when VLA-4 was blocked (21). Both these studies suggest a role for VLA-4 in CD18-independent neutrophil emigration from the capillaries to the airspaces.

To elucidate the role of VLA-4 in CD18-independent neutrophil emigration during bacterial pneumonia, we examined the expression of VLA-4 and VCAM-1 on neutrophils and endothelial cells during pneumonia, as well as the role of VLA-4 when the function of CD18 was blocked by either antibodies or by genetic deficiency. VLA-4 expression was measured on neutrophils obtained from the bone marrow, circulating blood, or BAL fluid in wild-type (WT) and CD18-deficient mice. VCAM-1 mRNA and protein expression was examined using reverse transcription-polymerase chain reaction (RT-PCR) and immunohistochemistry. The function of VLA-4 in neutrophil emigration and edema formation was examined during $S$. pneumoniae and E. coli pneumonias, where neutrophil emigration occurs through primarily CD18-independent and -dependent pathways, respectively. The function of VLA-4 was evaluated during blockade of CD18 using two distinct approaches. First, the effect of an anti-VLA-4 antibody was evaluated when CD18dependent emigration was blocked using an anti-CD18 antibody. Second, mice with both CD18-deficient and WT circulating neutrophils were generated by reconstituting the hematopoietic systems of lethally irradiated C57BL/6 mice with a mixture of fetal liver cells from WT and CD18-deficient mice. Recruitment of CD18-deficient neutrophils into the alveolar space during $S$. pneumoniae pneumonia was examined using these reconstituted mice after pretreatment with either anti-VLA-4 antibody or control IgG.

\section{METHODS}

\section{Protocol 1: VLA-4 Expression on Neutrophils}

CD18-deficient mice (22-24) and WT mice of the same genotype $(\mathrm{C} 57 \mathrm{BL} / 6 \times 129 / \mathrm{Sv})$, generously provided by Dr. Arthur L. Beaudet at Baylor College of Medicine, Houston, TX, were anesthetized and given an intratracheal instillation of $S$. pneumoniae $(50 \mu \mathrm{l} / 22 \mathrm{~g}$ body weight [bw] of $3.5 \times 10^{7}$ colony forming units [CFU]/ml suspension$5 \%$ colloidal carbon) as previously described (22, 23, 25-27). After 6 hours, lungs were lavaged with phosphate-buffered saline (PBS)-0.6 $\mathrm{mM}$ ethylenediamine tetraacetic acid (EDTA). Blood samples from infected and noninfected mice were obtained from the inferior vena cava, and bone marrow cells were obtained from the femurs. VLA-4 expression was measured by flow cytometry using fluoroscein isothiocyanate (FITC)-conjugated anti-murine VLA-4 mAb (R1-2, rat IgG2b, ; PharMingen, San Diego, CA) and a phycoerythrin (PE)-conjugated $\mathrm{mAb}$ against the murine granulocyte marker Gr-1 (RB6-8C5; PharMingen [28]). FITC-conjugated rat IgG2b,к (A95-1; PharMingen) was used as an isotype control for the anti-VLA-4 antibody.

\section{Protocol 2: mRNA and Protein Expression of VCAM-1 during Pneumonia}

Anesthetized C57BL/6 mice received intratracheal instillation of $S$. pneumoniae $\left(5.4 \times 10^{8} \mathrm{CFU} / \mathrm{ml}\right)$ or E. coli $\left(1.1 \times 10^{8} \mathrm{CFU} / \mathrm{ml}\right)$ as in Protocol 1. VCAM-1 mRNA and protein expression were studied 4 and 6 hours later, respectively. Pneumonic and uninfected lungs were either frozen in liquid nitrogen for total RNA extractions or were inflated with Tissue-Tek diluted 1:1 with PBS ( $0.8 \mathrm{ml} / \mathrm{lung})$ and frozen for immunohistochemistry.

\section{Protocol 3: The Effect of Anti-VLA-4 mAb on Acute Inflammation during Pneumonia}

To determine the role of VLA-4 in CD18-independent neutrophil emigration, two groups of C57BL/6 mice (8-12 weeks old, Harlan Spra-
gue-Dawley) were studied. Group 1 was given an intravenous injection of both anti-CD18 mAb (2E6, $2 \mathrm{mg} / \mathrm{kg}$; Endogen, Woburn, MA) and anti-VLA-4 mAb (PS/2; $4 \mathrm{mg} / \mathrm{kg}) 15$ minutes before bacterial instillation; Group 2 received anti-CD18 mAb (2E6; $2 \mathrm{mg} / \mathrm{kg})$ and rat $\mathrm{IgG}(4 \mathrm{mg} / \mathrm{kg})$ instead of the anti-VLA-4 mAb. The blocking antimouse VLA- 4 mAb PS/2 was kindly provided by Dr. C. Wayne Smith of Baylor College of Medicine. This antibody has been used for in vivo studies by many investigators (29-34).

Anesthetized mice received an intratracheal instillation of $S$. pneumoniae $\left(4.7 \pm 0.3 \times 10^{8} \mathrm{CFU} / \mathrm{ml}\right)$ or E. coli $\left(1.0 \pm 0.2 \times 10^{8} \mathrm{CFU} / \mathrm{ml}\right)$ suspension $(50 \mu \mathrm{l} / 22 \mathrm{~g} \mathrm{bw})$ as described in Protocol 1. Edema formation was measured using radioisotopes, and neutrophil emigration was quantitated morphometrically using histologic sections $(23,25-$ 27). Circulating leukocyte and neutrophil counts were measured in blood samples from the inferior vena cava.

\section{Protocol 4: Effect of Anti-VLA-4 mAb on CD18-Deficient Neutrophil Emigration}

The percentages of CD18-deficient neutrophils in the circulating and emigrated pools during $S$. pneumoniae pneumonia were compared using lethally irradiated and reconstituted mice whose bone marrows were producing both CD18-deficient and WT neutrophils as previously described (22). At least 2 weeks after cessation of antibiotics, mice received an intravenous injection of either rat $\mathrm{IgG}(4 \mathrm{mg} / \mathrm{kg})$ or anti-VLA-4 mAb (PS/2; $4 \mathrm{mg} / \mathrm{kg}) 15$ minutes before pneumonia was induced with $S$. pneumoniae $\left(1.1 \times 10^{8} \mathrm{CFU} / \mathrm{ml}\right)$. After 6 hours, the migration of CD11/CD18-deficient neutrophils into the airspaces was measured using immunocytochemistry (22).

\section{Statistics}

Data are presented as mean \pm standard error of the mean. One-way analyses of variance, Fisher's least-significant difference test, $t$ tests, and paired $t$ tests were used as appropriate.

\section{RESULTS}

\section{VLA-4 Expression on Neutrophils}

To determine whether VLA-4 expression increased on neutrophils that migrated using CD18-independent pathways, VLA-4 expression on neutrophils obtained from either peripheral blood or BAL fluid during $S$. pneumoniae pneumonia was estimated using flow cytometric analyses (Figure 1A). Labeling neutrophils from either WT or CD18-null mice with the isotype (rat IgG2b, $)$ control antibody yielded less than one-tenth the fluorescence intensity (ranged from undetectable to $0.2 \times$ mean channel fluorescence $[\mathrm{MCF}]$ ) observed with the antiVLA-4 antibody. In WT mice, instillation of $S$. pneumoniae did not induce any changes in VLA-4 expression on circulating neutrophils, but surprisingly, the neutrophils that emigrated into the BAL fluid in these same mice expressed less VLA-4 than the circulating neutrophils $(\mathrm{p}<0.05)$. In uninfected CD18-deficient mice, the circulating neutrophils expressed less VLA-4 than in WT mice $(\mathrm{p}<0.05)$. S. pneumoniae induced a further decrease in VLA-4 expression on circulating CD18-deficient neutrophils ( $\mathrm{p}<0.05$ ), but no additional decrease occurred upon migration of these neutrophils into the alveolar space (Figure 1A). There was no significant difference in VLA-4 expression on BAL neutrophils between WT and CD18-deficient mice.

Because VLA-4 expression on circulating neutrophils was decreased in CD18-deficient mice compared with WT mice without experimental infection, we compared the expression of VLA-4 on the bone marrow neutrophils. However, no difference in VLA-4 expression was observed (Figure 1B). Circulating neutrophils from these same CD18-deficient mice expressed less VLA-4 than those in the bone marrow (and less than circulating neutrophils from WT mice, confirming the observations in Figure 1A), whereas no decrease in VLA-4 was observed in circulating neutrophils compared with bone marrow neutrophils in WT mice. 
A

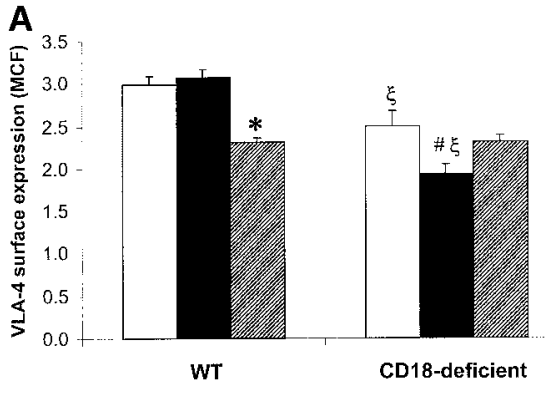

B

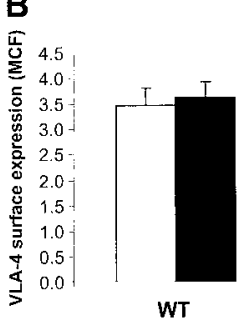

WT

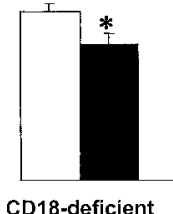

CD18-deficient

Figure 1. (A) VLA-4 expression on neutrophils obtained from blood or BAL fluid in uninfected mice or mice with S. pneumoniae-induced pneumonia for 6 hours. For flow cytometric analyses, heparinized blood was taken from the inferior vena cava, and erythrocytes were hypotonically lysed. Blood and BAL leukocytes were washed with fresh PBS-EDTA. Cells were stained with saturating concentrations of a FITC-conjugated mAb against murine VLA-4 (R1-2; PharMingen) and a $\mathrm{PE}$-conjugated $\mathrm{mAb}$ against the murine granulocyte marker Gr-1 (RB6-8C5; PharMingen) as previously described (28). Cells were then fixed with PBS containing $1 \%$ paraformaldehyde. Neutrophils were gated on a two-dimensional forward and side scattergram of the Coulter ELITE flow cytometer (Coulter Co., Miami, FL). Gr-1 bright cells had forward and side scatter characteristics consistent with granulocytes. The fluorescence intensity was measured for each sample by scoring 5,000 Gr-1 bright cells. The VLA-4 expression of neutrophils was represented by the mean channel fluorescence of 5,000 cells. The MCF values obtained when neutrophils from either WT or CD18-null mice were labeled with the isotype $(\mathrm{rat} \lg \mathrm{G} 2 \mathrm{~b}, \mathrm{k}$ ) control antibody ranged from undetectable to $0.2 \mathrm{MCF}$. In WT mice, VLA-4 expression was similar on neutrophils from the blood in uninfected (open bars) and S. pneumoniae-infected (closed bars) mice, whereas neutrophils that had emigrated in the alveolar spaces expressed less VLA-4 (shaded bars). CD18-deficient mice had significantly less VLA-4 expressed on circulating neutrophils with or without infection by S. pneumoniae when compared with WT mice. Emigration into the alveolar spaces did not induce a further reduction. $\mathrm{n}=3$ in each group. ${ }^{*} p<0.05$ compared with blood from mice with S. pneumoniae pneumonia. ${ }^{\#} p<0.05$ compared with blood from uninfected mice. ${ }^{\xi} p<0.05$ compared with WT mice of comparable group. (B) VLA-4 expression on neutrophils obtained from bone marrow or peripheral blood from mice without experimentally induced pneumonia. For flow cytometry analyses, bone marrow cells were collected by flushing the bone marrow cavity with $1.0 \mathrm{ml}$ of PBS containing $0.6 \mathrm{mM}$ EDTA. Blood and bone marrow cells were stained as described in A. VLA-4 expression on neutrophils from the bone marrow was similar in CD18-deficient and WT mice. Open bars: bone marrow. Closed bars: blood. $\mathrm{n}=3$ in each group. ${ }^{*} \mathrm{p}<0.05$ compared with bone marrow.

\section{Gene Expression of VCAM-1 during Bacterial Pneumonia}

Gene expression of VCAM-1, a ligand of VLA-4, was estimated in murine lungs with and without pneumonia using RTPCR at 4 hours after instillation of organisms (Table 1). Total RNA was isolated from lung homogenates using RNAzol B (Tel-Test, Friendswood, TX) and reverse transcribed, and the products were amplified using either of two sets of specific primers (A and B) for mouse VCAM-1 (35). $\beta$-Actin was am-

TABLE 1. VCAM-1 GENE EXPRESSION

\begin{tabular}{lccr}
\hline & \multicolumn{3}{c}{ Relative Intensity } \\
\cline { 2 - 4 } Primer Set & Uninfected & Streptococcus pneumoniae & Escherichia coli \\
\hline A & $1.00 \pm 0.05$ & $4.51 \pm 0.15^{*}$ & $11.69 \pm 0.61^{* \dagger}$ \\
B & $1.00 \pm 0.07$ & $1.28 \pm 0.19$ & $9.53 \pm 0.77^{* \dagger}$ \\
\hline
\end{tabular}

Anesthetized $\mathrm{C} 57 \mathrm{BL} / 6$ mice received intratracheal instillation of bacterial suspension $\left(50 \mu \mathrm{l} / 22 \mathrm{~g}\right.$ of body weight) containing S. pneumoniae $\left(5.4 \times 10^{8} \mathrm{CFU} / \mathrm{ml}\right)$ or $E$. coli $\left(1.1 \times 10^{8} \mathrm{CFU} / \mathrm{ml}\right)$ for 4 hours. Lungs were frozen immediately in liquid nitrogen and stored at $-80^{\circ} \mathrm{C}$ until total RNA extractions were performed. Total RNA was isolated from lung homogenates (Polytron aggregate; Kinematica, Lucerne, Switzerland) using RNAzol B (Tel-Test, Friendswood, TX) following manufacturer's instructions. RNA concentrations were determined by spectrophotometric absorption at $260 \mathrm{~nm}$. Total RNA $(2.5 \mu \mathrm{g})$ was reverse transcribed with $200 \mathrm{U}$ of Moloney murine leukemia virus reverse transcriptase and $500 \mathrm{ng}$ of oligo(dT)12-18 primer (Gibco BRL, Gaithersburg, MD). The reverse transcribed products $(1 \mu \mathrm{l})$ were amplified using two sets of specific primers (A and B) for mouse VCAM-1 (29). Both sets of primers represent an extracellular region of VCAM-1. $\beta$-Actin was used as a housekeeping gene. The PCR amplifications were perfomed in a $50 \mu \mathrm{l}$ reaction volume containing $10 \mathrm{mM}$ Tris-HC1 $(\mathrm{pH} 9.0), 50$ $\mathrm{mM} \mathrm{KCl}, 1.5 \mathrm{mM} \mathrm{MgCl}_{2}, 0.2 \mathrm{mM}$ dNTPs, and $1.5 \mathrm{U}$ of Taq polymerase (Pharmacia Biotech, Piscataway, NI) in PTC-100TM programmable thermal controller (MJ Research, Inc., Watertown, MA) under conditions determined in earlier experiments to be optimal for exponential amplification. In most experiments, cycling profiles included an initial denaturation at $94^{\circ} \mathrm{C}, 5$ minutes; 5 minutes annealing at $60^{\circ} \mathrm{C}$; followed by 35 cycles of $94^{\circ} \mathrm{C}$ for 45 seconds, $60^{\circ} \mathrm{C}$ for 45 seconds, and $72^{\circ} \mathrm{C}$ for 90 seconds for denaturing, annealing, and extension, respectively. The cycling was completed with a final incubation at $72^{\circ} \mathrm{C}$ for 10 minutes. The primers used were as follows: VCAM-1, primer pair A: sense, 5'-CAA CGA TCT CTG TAC ATC CC-3'; antisense, 5'-AGA GGC TGT ACA CTC TGC CT-3'. VCAM-1, primer pair B: sense, 5'-AAG GAT CCG GTA CCA AGC AGA GAC TTG AAA TGC C-3'; antisense, 5' -CCC TTG AAC AGA TCA ATC TCC-3'. $\beta$-Actin: sense, 5'-GTG GGC CGC TCT AGG CAG-3'; antisense, 5'-CTT TGA TGT CAC GCA TTT C-3'. Amplified DNAs were resolved by means of agarose gel electrophoresis and stained with ethidium bromide. Polaroid photographs with ultraviolet exposure were taken, and the images were converted into numerical data by quantitative densitometry with Scion ImagePC software (Scion, Frederick, MD).

${ }^{*} p<0.01$ compared with uninfected control.

${ }^{\dagger} \mathrm{p}<0.01$ compared with S. pneumoniae pneumonia. plified in separate reactions to control for RNA content. PCR products were revealed by ethidium bromide staining and quantified by densitometry.

VCAM-1 was amplified using two sets of primer pairs, both of which amplify the extracellular region of VCAM-1. Primer pair A amplified mRNA that coded for a portion of Ig domain 2 through domains 3-5. Primer pair B amplified mRNA coding for regions near the carboxyl terminus including the signal peptide, Ig domain 1, and part of domain 2. Both sets of primer pairs gave a single PCR product of the expected size and sequence. After 4 hours, E. coli induced a greater increase than did $S$. pneumoniae ( $\mathrm{p}<0.01$ ), no matter which primer set was used. RT-PCR using primer set A showed increased VCAM-1 mRNA expression in $S$. pneumoniae compared with uninfected lung tissue but less than in E. coli pneumonia, and RT-PCR using primer set B showed increased VCAM-1 mRNA expression only in E. coli pneumonia (Table 1).

\section{Localization of VCAM-1 Protein Expression}

Immunohistochemistry was performed to localize VCAM-1 expression as previously described (25) using either the rat antiVCAM-1 429 monoclonal antibody (Endogen) or rat IgG and

TABLE 2. VCAM-1 PROTEIN EXPRESSION ON ARTERIOLES AND VENULES (NUMBER OF VESSELS PER 10 FIELDS AT $\times 200$ MAGNIFICATION)

\begin{tabular}{lcc}
\hline & Brightly Staining & Lightly Staining \\
\hline Uninfected & $21.7 \pm 2.9$ & $19.7 \pm 3.0$ \\
Streptococcus pneumoniae & $20.2 \pm 3.2$ & $22.3 \pm 1.6$ \\
Escherichia coli & $15.8 \pm 2.5$ & $19.3 \pm 2.1$ \\
\hline
\end{tabular}

Immunohistochemistry was performed to localize VCAM-1 expression in mice without infection or with S. pneumoniae or E. coli pneumonias for 6 hours as previoulsy described (25). In brief, frozen sections measuring 4-6 $\mu \mathrm{m}$ were cut, fixed, blocked with avidin followed by biotin and goat IgG, in methanol: acetone (1:1), and incubated with either rat anti-VCAM-1 monoclonal antibody (Clone \#429; PharMingen) or rat IgG. After washing, the sections were incubated with biotinylated goat anti-rat lgG polyclonal antibody, followed by streptavidin-labeled alkaline phosphatase. The complexes were identified by incubating with a substrate combined with new fuchsin. After coverslipping, VCAM-1 expression on capillaries, arteries, and veins was evaluated at $\times 600$ magnification (see ResulTs). VCAM-1 expression on arterioles and venules was quantitated by counting the number of vessels that stained either brightly or lightly per 10 fields at $\times 200$ magnification. 
a streptavidin-alkaline phosphatase detection system. VCAM-1 expression on capillaries, arteries, and veins was evaluated at $\times 600$ magnification. Expression on arterioles and venules was quantitated by counting the number of vessels that stained either brightly or lightly per 10 fields at $\times 200$ magnification.

In uninfected lungs, VCAM-1 was detectable only on arterial and venous endothelium. No capillary endothelium expressed VCAM-1. Neither $S$. pneumoniae nor E. coli induced VCAM-1 expression on the capillary endothelium within 6 hours. In addition, neither organism induced an increase in staining on the arteriolar or the venular endothelium (Table 2). Many of these small vessels and virtually all the larger vessels stained brightly, making an increase hard to detect.

\section{Effect of Anti-VLA-4 mAb on Acute Inflammation during Pneumonia}

These studies used the blocking rat anti-mouse VLA-4 monoclonal antibody PS/2, a well-described antibody that has been used previously in vivo to block VLA-4-mediated responses. For example, this antibody protects against contact hypersensitivity to 2,4-dinitrofluorobenzene or oxazolone $(29,30)$, inhibits ovalbumin delayed-type hypersensitivity (30), induces immunosuppression to cardiac allografts and soluble antigens (31), inhibits eosinophil recruitment in ovalbumin-sensitized and challenged lungs (31) and in allergic conjunctivitis (33), and prevents mast cell emigration into the intestine in response to the nemotode Trichinella spiralis (34). After pretreatment with anti-CD18 antibody and either the anti-VLA-4 antibody $\mathrm{PS} / 2$ or nonspecific rat IgG, C57BL/6 mice were given intratracheal instillations of either $S$. pneumoniae or E. coli. Edema and neutrophil emigration were evaluated 6 hours later. Edema formation was measured by quantitating the accumulation of extravascular albumin using ${ }^{125} \mathrm{I}$-labeled and ${ }^{51} \mathrm{Cr}$-labeled red blood cells (RBC) as previously described $(23,25-27)$ and expressed as microliters per gram bw. Emigrated neutrophils were quantified by morphometry in histologic sections of car-

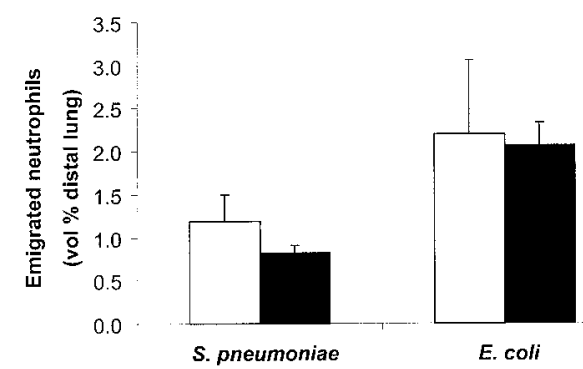

Figure 2. Neutrophil emigration into the alveolar spaces in mice with 6-hour S. pneumoniae or E. coli pneumonia. Mice received an intravenous injection of anti-CD18 antibody and either rat IgG (open bars) or anti-VLA-4 mAb (closed bars) 15 minutes before instillation of the organisms. After 6 hours, the lungs were removed and fixed using intratracheal instillation of $6 \%$ glutaraldehyde at $22 \mathrm{~cm} \mathrm{H}_{2} \mathrm{O}$. Emigrated neutrophils were quantified by morphometry in histologic sections (23). Carbon black-containing lung regions were embedded in paraffin, and 5- to 7- $\mu \mathrm{m}$ thick sections were cut and stained with hematoxylin and eosin. A counting grid $\left(10 \times 10\right.$, covering $70,000 \mu \mathrm{m}^{2}$ of the magnified field) was reflected onto the field of view at $\times 1,000$ magnification using a drawing tube. Randomly selected fields of pneumonic peripheral lung where alveolar macrophages contained carbon particles were examined. Regions that contained noncapillary blood vessels, bronchioles, or larger airways were not examined. A total of 10 grids (1,000 points) was counted for each lung, and neutrophil emigration was expressed as the fraction of distal lung tissue that was occupied by intra-alveolar (emigrated) neutrophils. Blockade of VLA-4 did not alter the CD18-independent emigration of neutrophils in response to either stimulus. $\mathrm{n}=5$ in each group. bon black-containing lung regions using point counting techniques as previously described $(23,25,26)$ and expressed as the fraction of distal lung tissue that was occupied by intraalveolar (emigrated) neutrophils.

Blockade of VLA-4 by PS/ 2 had no significant effect on the CD18-independent component of neutrophil emigration induced by either S. pneumoniae or E. coli (Figure 2). Similarly, blockade of VLA-4 had no effect on the formation of pulmonary edema during $S$. pneumoniae or E. coli pneumonia (Figure 3).

Circulating leukocyte and neutrophil counts in blood samples obtained from the inferior vena cava are shown in Table 3 . The leukocyte and neutrophil counts were greater in mice pretreated with both anti-CD18 and anti-VLA-4 mAb than in mice pretreated with only the anti-CD18 mAb after 6 hours $E$. coli pneumonia $(\mathrm{p}<0.05)$. The anti-VLA-4 mAb did not induce a significant difference in leukocyte or neutrophil count in $S$. pneumoniae pneumonia.

\section{Effect of Anti-VLA-4 mAb on CD18-Deficient Neutrophil Emigration}

To further evaluate the role of VLA-4 in CD18-independent neutrophil emigration, a second approach was taken where mice with bone marrows producing both CD18-deficient and WT neutrophils were treated with the anti-VLA-4 mAb. This approach is more likely to detect a small contribution of VLA-4 because the emigration of CD18-null and WT neutrophils are compared in the same pneumonia within one mouse, so that the physiologic parameters and the inflammatory milieu are identical for cells of each genotype. The hematopoietic systems of lethally irradiated C57BL/6 host mice were reconstituted by injection of a mixture of CD18-deficient (20\%) and WT (80\%) 14-day fetal liver cells as previously described (22). At least 2 weeks after cessation of antibiotics, pneumonia was induced by intratracheal instillation of $S$. pneumoniae $(50 \mu \mathrm{l} /$ $22 \mathrm{~g}$ bw of a suspension containing $\left.1.1 \times 10^{8} \mathrm{CFU} / \mathrm{ml}\right)$. Mice received intravenous injection of either rat $\mathrm{IgG}(4 \mathrm{mg} / \mathrm{kg})$ or

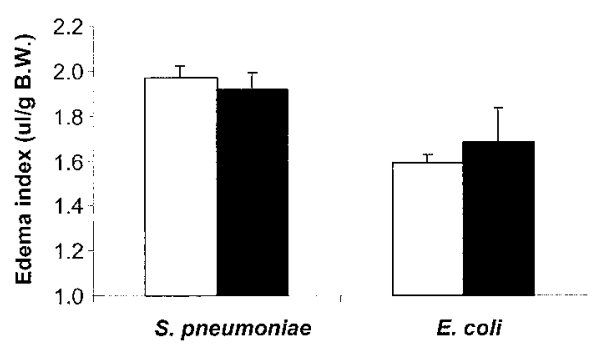

Figure 3. Edema formation during 6-hour S. pneumoniae or E. coli pneumonia. Mice received an intravenous injection of anti-CD18 antibody and either rat IgG (open bars) or anti-VLA-4 mAb (closed bars) combined with ${ }^{125}$ I-labeled human albumin $(0.1 \mu \mathrm{Ci}$ /mouse; Mallinckrodt Medical Inc., Hazelwood, MO) to measure edema. Murine RBC labeled with ${ }^{51} \mathrm{Cr}(0.3 \mu \mathrm{Ci} /$ mouse; DuPont-NEN, Boston, MA) were injected 2 minutes before the end of the study to measure the intravascular blood volume. At 6 hours, the lungs were removed, blood samples were obtained from the inferior vena cava, and levels of radioactivity were measured in a $\gamma$ counter. Edema formation in lungs was evaluated by quantitating the accumulation of extravascular albumin as previously described $(23,26,27)$. Blood and plasma volumes of lungs were determined from ${ }^{51} \mathrm{Cr}$-RBC and ${ }^{125} \mathrm{I}$-albumin counts, respectively. Pulmonary edema (extravascular albumin in the lung) was calculated as total lung albumin content minus intravascular lung albumin content. Intravascular lung albumin content was calculated from the pulmonary blood volume and the hematocrit for each animal. Pulmonary edema was expressed as $\mu \mathrm{l} / \mathrm{g}$ bw. Blockade of VLA-4 did not alter the formation of edema. $n=5$ in each group. 
TABLE 3. CIRCULATING LEUKOCYTE AND NEUTROPHIL COUNTS DURING BACTERIAL PNEUMONIA

\begin{tabular}{|c|c|c|}
\hline & Leukocytes $\left(\times 10^{6} / \mathrm{ml}\right)$ & Neutrophils $\left(\times 10^{6} / \mathrm{ml}\right)$ \\
\hline & Rat IgG & Rat IgG \\
\hline treptococcus pneumoniae & $2.76 \pm 0.441 .96 \pm 0.40$ & $1.62 \pm 0.220 .99 \pm$ \\
\hline Escherichia coli & $1.47 \pm 0.264 .06 \pm$ & $0.43 \pm 0.060 .97$ \\
\hline \multicolumn{3}{|c|}{$\begin{array}{l}\text { White blood cells were counted using a hemocytometer, and differentials were de- } \\
\text { termined using blood smears stained with Leukostat (Fischer Scientific, Pittsburgh, PA). } \\
\text { In mice with S. pneumoniae pneumonia, PS } / 2 \text { administration had no significant effects } \\
\text { on the circulating leukocyte and neutrophil counts in blood samples from the inferior } \\
\text { vena cava obtained } 6 \text { hours after instillation. In contrast, both leukocyte and neutrophil } \\
\text { counts were greater in PS/2-treated mice than in mice given rat IgG at } 6 \text { hours after in- } \\
\text { stillation of } E \text {. coli. } \\
\quad{ }^{*} \mathrm{p}<0.05 \text {. } \\
\text { Values are means } \pm \text { SEM, } \mathrm{n}=5 \text {. }\end{array}$} \\
\hline
\end{tabular}

anti-VLA-4 mAb (PS/2; 4 mg/kg) 15 minutes before bacterial instillation. After 6 hours, BAL was performed as described in Protocol 1. Leukocyte and neutrophil counts in blood and BAL fluid were determined. The percentage of CD18-deficient neutrophils was assessed for each blood and BAL sample by scoring 300 neutrophils in cytocentrifuged preparations as either positive or negative for CD11b/CD18 expression using immunocytochemical staining for CD11b (M1/70; PharMingen), as previously described (22).

In the mice pretreated with rat $\mathrm{IgG}$, the percentage of CD18-deficient neutrophils did not differ between the circulating blood and the BAL fluid (Figure 4), indicating that the CD18deficient neutrophils showed no defect in emigration toward this stimulus. In contrast, the percentage of CD18-deficient neutrophils was slightly but significantly decreased in the BAL fluid compared with the circulating blood when the mice were given PS/ 2 to block VLA-4 $(\mathrm{p}<0.01)$. These observations indicate that the CD18-deficient neutrophils were not able to migrate as well as the CD18-replete neutrophils when VLA-4 was blocked, suggesting a small but significant role for VLA-4 in CD18-independent emigration. There were no significant differences between the groups in neutrophil counts in BAL fluid (rat IgG, $1.36 \pm 0.26 \times 10^{6} / \mathrm{ml}$; PS $/ 2,0.58 \pm 0.05 \times 10^{6} / \mathrm{ml}$; $\mathrm{p}=0.099)$ or blood (rat IgG, $1.66 \pm 0.26 \times 10^{6} / \mathrm{ml} ; \mathrm{PS} / 2,1.89 \pm$ $\left.0.50 \times 10^{6} / \mathrm{ml}\right)$.

\section{DISCUSSION}

This study examined the effect of pulmonary infection on VLA-4 expression in neutrophils and on VCAM-1 expression in pulmonary endothelial cells, as well as the functional role of VLA-4 in CD18-independent neutrophil emigration. VLA-4 expression on emigrated neutrophils decreased during $S$. pneumoniae pneumonia. VCAM-1 mRNA was increased many fold in E. coli pneumonia by 4 hours, but not at all or only slightly during $S$. pneumoniae pneumonia. In pneumonia induced by either organism, VCAM-1 protein expression did not occur on capillaries, which are the major site of neutrophil emigration (36-40). Blockade of both VLA-4 and CD18 did not decrease neutrophil emigration further than blockade of CD18 alone. More sensitive studies comparing the migration of CD18-deficient neutrophils in mice whose bone marrows were producing both CD18-deficient and WT neutrophils showed that a small fraction of CD18-independent neutrophil emigration required VLA-4. These studies suggest that whereas the inflammatory response results in alterations of VLA-4 and VCAM-1 expression, only a very small component of CD18-independent adhesion required VLA-4, and VLA-4/CD18-independent pathways play an important role in neutrophil emigration during bacterial pneumonia.

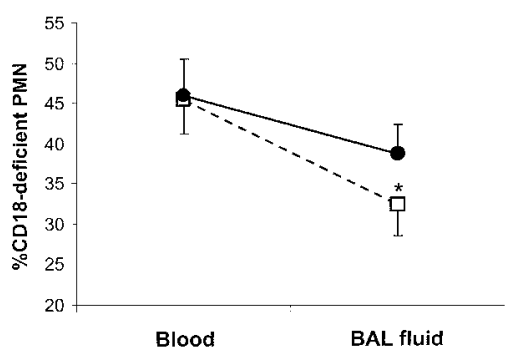

Figure 4. Percentage of CD18-deficient neutrophils in blood and BAL fluid obtained after 6-hour S. pneumoniae pneumonia. The hematopoietic systems of lethally irradiated C57BL/ 6 host mice were reconstituted by injection of a mixture of CD18-deficient and WT fetal liver cells as previously described (22). CD18deficient mice and WT mice of the similar randomly mixed genetic background $(\mathrm{C} 57 \mathrm{BL} / 6 \times 129 / \mathrm{Sv})$ were mated with the like genotype, and fetuses were collected at 14 days of gestation. Single-cell suspensions were prepared from WT and CD18-deficient fetal livers, and mixtures (20\% CD18-deficient; $80 \%$ WT) of $2 \times 10^{6}$ total cells were injected intravenously into host mice that had received radiation doses of 800 and 400 rad, separated by 3 hours, from a ${ }^{137} \mathrm{Cs}$ source. After transplantation, mice received trimethoprim-sulfamethoxazole in their drinking water for 4 weeks and were housed in a full barrier. At least 2 weeks after cessation of antibiotics, pneumonia was induced with S. pneumoniae $\left(1.1 \times 10^{8} \mathrm{CFU} / \mathrm{ml}\right)$ as described previously. Mice received intravenous injection of either rat $\mathrm{lgG}(4 \mathrm{mg} / \mathrm{kg}$, squares) or anti-VLA-4 $\mathrm{mAb}(\mathrm{PS} / 2 ; 4 \mathrm{mg} / \mathrm{kg}$, circles) 15 minutes before bacterial instillation. After 6 hours, BAL was performed. Heparinized blood was obtained from the inferior vena cava, and erythrocytes were hypotonically lysed. Blood and BAL leukocytes were washed with fresh PBS-EDTA. Leukocytes and neutrophil counts in blood and BAL fluid were determined. For immunohistochemical analyses, blood and BAL cells were cytocentrifuged onto glass slides, allowed to air dry, and then fixed with acetone:methanol $(1: 1)$. Slides were treated with biotinylated rat $\mathrm{mAb}$ against murine CD11b (M1/70; PharMingen), and M1/70 was visualized using a streptavidin-alkaline phosphatase detection system. Control slides demonstrated no staining of cells when nonspecific rat IgG replaced $M 1 / 70$. Slides were counterstained with hematoxylin, and the percentage of CD18-deficient neutrophils was assessed for each sample by scoring 300 polymorphonuclear cells as either positive or negative for $C D 11 \mathrm{~b} / C D 18$ expression (22). The data show that the percentage of CD18-deficient neutrophils in the blood and the BAL fluid was similar when the function of VLA-4 was not inhibited. Blockade of VLA-4 did induce a small but significant decrease in the emigration of CD18-deficient cells. $\mathrm{n}=5$ in each group. ${ }^{*} \mathrm{p}<0.05$ compared with peripheral blood. Squares: mice treated with anti-VLA-4 antibody 15 minutes before instillation of organisms. Circles: mice pretreated with rat $\lg G$ (control animals).

In contrast to the results of Kubes and colleagues (15), we observed no increase in VLA-4 expression on emigrated neutrophils and in fact observed a decrease as compared with circulating neutrophils. Although this could be due to decreased production of VLA-4, neutrophils have not been found to produce this molecule after they have left the circulation, making this possibility unlikely. Alternatively, this molecule may be cleaved during the inflammatory process, either during the process of emigration, as suggested by the observations in WT mice, or while still in the circulation, as suggested by the observations in CD18-deficient mice. The fact that the CD18deficient mice have lower expression of VLA-4 on circulating neutrophils in the absence of $S$. pneumoniae does not exclude an effect of the inflammatory process because these mice have spontaneous infections as manifested by skin lesions and high circulating neutrophil counts $(23,24)$. The observation that the expression of VLA-4 on bone marrow neutrophils was similar in CD18-deficient and WT mice indicates that the reduction in VLA-4 expression on circulating neutrophils in CD18-deficient mice was not a direct effect of deletion of CD18 gene transcription.

In the present study, the addition of anti-VLA-4 mAb to anti-CD18 mAb failed to further inhibit neutrophil emigration during E. coli and S. pneumoniae pneumonias. Thus, blocking 
VLA-4 did not inhibit the CD18-independent component of neutrophil emigration in either $E$. coli pneumonia, where the CD18-independent component comprises only $30 \%$ of emigration, or in S. pneumoniae pneumonia, where all neutrophil emigration occurs through CD18-independent pathways. In addition to mediating the adhesion and emigration of circulating leukocytes, VLA-4 may contribute to other local and systemic responses to inflammatory stimuli. For example, studies by Henderson and colleagues (41) demonstrated in an airway hypersensitivity study that VLA-4 was required for cytokine release in the lungs. In the present studies, intravenous administration of blocking VLA-4 antibodies had no effect on edema accumulation during either E. coli or S. pneumoniae pneumonias. These data suggest that VLA-4-mediated cell interactions are not required for the signaling events resulting in local plasma extravasation.

Because the role of VLA-4 might be too small to measure differences in these complex parameters of inflammation, studies were performed using mice whose bone marrows expressed both CD18-deficient and WT neutrophils. This approach will likely detect a subtler role for VLA-4 in neutrophil emigration, partly because each mouse serves as its own control and the CD18 null and WT genotypes can be compared in the same hemodynamics and inflammatory milieu. The percentages of CD18deficient neutrophils in the circulating and emigrated pools were compared during $S$. pneumoniae pneumonia. In mice pretreated with rat $\mathrm{IgG}$, similar percentages of CD18-deficient neutrophils were observed in the blood and BAL fluid 6 hours after intratracheal instillation of $S$. pneumoniae, indicating that neutrophil emigration induced by $S$. pneumoniae was CD18independent. This observation is consistent with our previous experiments, which also revealed that the percentage of CD18deficient neutrophils in the BAL fluid was significantly smaller than in the peripheral blood in E. coli LPS pneumonia, a stimulus known to elicit CD18-dependent neutrophil emigration $(6,7,22)$.

However, in mice pretreated with anti-VLA-4 mAb, the percentage of CD18-deficient neutrophils in BAL neutrophils was significantly lower than the percentage in circulating neutrophils, suggesting that emigration of the CD18-deficient population was partially blocked by the anti-VLA-4 mAb. These data suggest that a small portion of CD18-independent emigration may be mediated through VLA-4, but at least one CD18- and VLA-4-independent pathway of neutrophil emigration exists and is functional in the lungs. An alternative explanation might be that CD18-null neutrophils use alternative pathways that are partially mediated by VLA-4, whereas WT neutrophils do not. In fact, observations in CD18-null mice support usage of alternative pathways $(23,25)$. However, these reconstituted mice have a large fraction of WT neutrophils that prevent the neutrophilia, the extramedullary hematopoiesis, infectious skin ulcerations, and other aspects of the CD18 null phenotype (23, 24,42 ), making the use of alternative pathways by the CD18null neutrophils less likely. Furthermore, there was no increase in VLA-4 expression in CD18-null neutrophils (Figure 1B). Therefore, we interpret these data to suggest that a small fraction of CD18-independent emigration in WT mice may occur through VLA-4-mediated pathways, but CD18-independent, VLA-4independent pathways clearly contribute.

These observations are consistent with those of Li and colleagues (43), who demonstrated that LPS-induced neutrophil emigration at later (18 hours) time points was partially inhibited by about $30 \%$ when rats were pretreated with antibodies against both CD18 and VLA-4. Previous reports also suggested that blocking VLA-4 during adjuvant-induced arthritis did not completely inhibit CD18-independent neutrophil emi- gration, although the contribution of VLA-4 appeared larger than in the lungs $(17,19)$. The studies by Ridger and colleagues (20) and by Burns and colleagues (21) both demonstrate a larger role for VLA-4 in CD18-independent emigration in response to $\mathrm{KC}$ and to LPS, respectively, compared with our studies of E. coli- and S. pneumoniae-induced emigration. However, both studies clearly show a component of emigration that does not require CD11/CD18 or VLA-4, and measures about $30 \%$ of KC-induced neutrophil emigration (20) and about $40 \%$ of LPS-induced emigration (21). Furthermore, the studies by Ridger and colleagues suggest that the role VLA-4 is playing is not in neutrophil-endothelial adhesion but rather in neutrophil emigration through the interstitium, leading to the postulate that VLA-4-fibronectin interactions contribute to neutrophil emigration (20). Taken together, all these studies suggest that the adhesion molecules mediating CD11/CD18independent interactions between neutrophils and pulmonary capillary endothelial cells remain unclear. In addition, the roles of VLA-4 in mediating either neutrophil-matrix or neutrophil-endothelial cells depend on the inflammatory stimulus. We are particularly interested in these bacterial stimuli, as they represent clinically important, albeit complex, pathogens.

At 4 hours after instillation of organisms, VCAM-1 gene expression as evaluated using RT-PCR appeared greater in $E$. coli pneumonia, which elicits CD18-dependent emigration, than in $S$. pneumoniae pneumonia, which elicits CD18-independent emigration. This result is compatible with a previous report describing increased production of VCAM-1 on endothelial cells by IL- 1 or TNF- $\alpha$ as early as 4 hours $(44,45)$. E. coli induces production of IL- 1 and TNF- $\alpha$, as well as the expression of ICAM-1 on the pulmonary capillary endothelium (46). Immunohistochemistry demonstrated that the endothelial cells producing VCAM-1 were not lining capillaries, the major site of neutrophil emigration (36-40). In fact, the immunohistochemistry did not demonstrate any site of increased protein production. Arteries, arterioles, venules, and veins showed VCAM-1 expression in uninfected murine lungs, and the highly sensitive but nonquantitative nature of immunohistochemistry may preclude detection of an increase at these sites. Alternatively, the increased transcription observed by RT-PCR may not lead to increased VCAM-1 protein expression. In light of the absence of VCAM-1 expression by the capillaries, the lack of a functional role for VLA-4 in acute neutrophil emigration is not surprising, and the small role observed in $S$. pneumoniae pneumonia may be due to a requirement for this molecule in the rolling or adhesion of the few neutrophils that migrate through arterioles or venules. This increased VCAM-1 mRNA may also function later in the course of the pneumonia, perhaps in the recruitment of monocytes or lymphocytes.

CD18-deficient mice have 5- to 30 -fold increases in circulating neutrophil counts and 3- to 5-fold increases in neutrophil emigration during pneumonia $(23,24)$. The molecules mediating this emigration remain enigmatic. The decrease in VLA-4 expression on circulating neutrophils in CD18-deficient mice compared with WT suggests that VLA-4 is not compensating for the deficiency of CD18. This conclusion is also supported by the lack of an increase in VLA-4 expression in neutrophils within the bone marrow. Finally, the data addressing the function of this molecule suggest that VLA-4 mediates only a small fraction of CD18-independent adhesion, although no studies were performed in CD18-deficient mice to directly test this hypothesis.

In summary, these studies show that the expression of VLA-4 on neutrophils is decreased during CD18-independent emigration induced by $S$. pneumoniae, that VCAM-1 is not upregulated on pulmonary capillaries in $S$. pneumoniae or $E$. 
coli pneumonia, and that VLA-4-mediated adhesion accounts for only a small fraction of neutrophils that emigrate through CD18-independent pathways. The molecules that mediate CD18-independent, VLA-4-independent neutrophil emigration remain to be determined.

Acknowledgment: The authors thank Sabrina D. Bhagwan and Amy Imrich for technical assistance and Virginia Ehrbar for preparing the manuscript. They are grateful to Dr. Arthur L. Beaudet for providing CD18-deficient mice and to Dr. C. Wayne Smith for providing the PS/2 antibodies and for many interesting discussions.

\section{References}

1. Hogg JC, Doerschuk CM. Leukocyte traffic in the lung. Annu Rev Physiol 1995;57:97-114.

2. Christopher RA, Guan JL. To move or not: how a cell responds. Int J Mol Med 2000;5:575-581.

3. Harris ES, McIntyre TM, Prescott SM, Zimmerman GA. The leukocyte integrins. J Biol Chem 2000;275:23409-23412.

4. Giancotti FG, Ruoslahti E. Integrin signaling. Science 1999;285:10281032.

5. Harlan JM, Winn RK, Vedder NB, Doerschuk CM, Rice CL. In vivo models of leukocyte adherence to endothelium. In: Harlan JM, Liu DY, editors. Adhesion: its role in inflammatory disease. New York: Freeman and Co.; 1992. p. 117-150.

6. Doerschuk CM, Mizgerd JP, Kubo H, Qin L, Kumasaka T. Adhesion molecules and cellular biomechanical changes in acute lung injury: Giles F. Filley Lecture. Chest 1999;116:37S-43S.

7. Doerschuk CM. Leukocyte trafficking in alveoli and airway passages. Respir Res 2000;1:136-140.

8. Lobb RR, Pepinsky B, Leone DR, Abraham WM. The role of $\alpha 4$ integrins in lung pathophysiology. Eur Respir J 1996;9:104S-108S.

9. Morimoto C, Iwata S, Tachibana K. VLA-4-mediated signaling. Curr Top Microbiol Immunol 1998;231:1-22.

10. Lobb RR, Hemler ME. The pathophysiologic role of $\alpha 4$ integrins in vivo. J Clin Invest 1994:94:1722-1728.

11. Johnston B, Chee A, Issekutz TB, Ugarova T, Fox-Robichaud A, Hickey MJ, Kubes P. $\alpha_{4}$ Integrin-dependent leukocyte recruitment does not require VCAM-1 in a chronic model of inflammation. J Immunol 2000;164:3337-3344.

12. Schneider T, Issekutz TB, Issekutz AC. The role of $\alpha 4$ (CD49d) and $\beta 2$ (CD18) integrins in eosinophil and neutrophil migration to allergic lung inflammation in the Brown Norway rat. Am J Respir Cell Mol Biol 1999;20:448-457.

13. Hojo M, Maghni K, Issekutz TB, Martin JG. Involvement of $\alpha-4$ integrins in allergic airway responses and mast cell degranulation in vivo. Am J Respir Crit Care Med 1998;158:1127-1133.

14. Abraham WM, Gill A, Ahmed A, Sielczak W, Lauredo IT, Botinnikova Y, Lin K-C, Pepinsky B, Leone DR, Lobb RR, et al. A small-molecule, tight binding inhibitor of the integrin $\alpha_{4} \beta_{1}$ blocks antigen-induced airway responses and inflammation in experimental asthma in sheep. Am J Respir Crit Care Med 2000;162:603-611.

15. Kubes P, Niu X-F, Smith CW, Kehrli ME Jr, Reinhardt PH, Woodman RC. A novel $\beta_{1}$-dependent adhesion pathway on neutrophils: a mechanism invoked by dihydrocytochalasin $B$ or endothelial transmigration. FASEB J 1995;9:1103-1111.

16. Reinhardt PH, Elliott JF, Kubes P. Neutrophils can adhere via $\alpha 4 \beta 1$ integrin under flow conditions. Blood 1997;89:3837-3846.

17. Issekutz TB, Miyasaka M, Issekutz AC. Rat blood neutrophils express very late antigen 4 and it mediates migration to arthritic joint and dermal inflammation. J Exp Med 1996;183:2175-2184.

18. Birner U, Issekutz TB, Issekutz AC. The role of selectins in VLA-4 and CD18-independent neutrophil migration to joints of rats with adjuvant arthritis. Eur J Immunol 1999;29:1094-1100.

19. Issekutz AC, Ayer L, Miyasaka M, Issekutz TB. Treatment of established adjuvant arthritis in rats with monoclonal antibody to CD18 and very late activation antigen-4 integrins suppresses neutrophil and T-lymphocyte migration to the joints and improves clinical disease. Immunology 1996;88:569-576.

20. Ridger VC, Wagner BE, Wallace WAH, Hellewell PG. Differential effects of CD18, CD29, and CD49 integrin subunit inhibiton on neutrophil migration in pulmonary inflammation. J Immunol 2001;166:3484-3490.

21. Burns JA, Issekutz TB, Yagita H, Issekutz AC. The $\alpha 4 \beta 1$ (very late antigen (VLA)-4, CD49d/CD29) and $\alpha 5 \beta 1$ (VLA-5, CD49e/CD29) integrins mediate $\beta 2(\mathrm{CD} 11 / \mathrm{CD} 18)$ integrin-independent neutrophil re- cruitment to endotoxin-induced lung inflammation. J Immunol 2001; 166:4644-4649.

22. Mizgerd JP, Horwitz BH, Quillen HC, Scott ML, Doerschuk CM. Effects of CD18 deficiency on the emigration of murine neutrophils during pneumonia. J Immunol 1999;163:995-999.

23. Mizgerd JP, Kubo H, Kutkoski GJ, Bhagwan SD, ScharffetterKochanek K, Beaudet AL, Doerschuk CM. Neutrophil emigration in the skin, lungs, and peritoneum: different requirements for CD11/ CD18 revealed by CD18-deficient mice. J Exp Med 1997;186:1357-1364.

24. Scharfetter-Kochanek J, Lu H, Norman K, van Nood N, Munoz F, Grabbe S, McArthur M, Lorenzo I, Kaplan S, Ley K, et al. Spontaneous skin ulceration and defective T cell function in CD18 null mice. $J$ Exp Med 1998;188:119-126.

25. Qin L, Quinlan WM, Doyle NA, Graham L, Sligh JE, Takei F, Beaudet AL, Doerschuk CM. The roles of CD11/CD18 and ICAM-1 in acute Pseudomonas aeruginosa-induced pneumonia in mice. J Immunol 1996; 157:5016-5021.

26. Bullard DC, Qin L, Lorenzo I, Quinlan WM, Doyle NA, Vestweber D, Doerschuk CM, Beaudet AL. P-selectin/ICAM-1 double mutant mice: acute emigration of neutrophils into the peritoneum is completely absent but is normal into pulmonary alveoli. J Clin Invest 1995; 95:1782-1788

27. Doerschuk CM, Quinlan WM, Doyle NA, Bullard DC, Vestweber D, Jones ML, Takei F, Ward PA, Beaudet AL. The roles of P-selectin and ICAM-1 in acute lung injury as determined using anti-adhesion molecule antibodies and mutant mice. J Immunol 1996;157:4609-4614.

28. Mizgerd JP, Bullard DC, Hicks MJ, Beaudet AL, Doerschuk CM. Chronic inflammatory disease alters adhesion molecule requirements for acute neutrophil emigration in mouse skin. J Immunol 1999;162: 5444-5448.

29. Chisholm PL, Williams CA, Lobb RR. Monoclonal antibodies to the integrin $\alpha 4$ subunit inhibit the murine contact hypersensitivity response. Eur J Immunol 1993;23:682-688.

30. Haworth D, Rees A, Alcock PJ, Wood LJ, Dutta AS, Gormley JJ, Jones HB, Jamieson A, Reilly CF. Anti-inflammatory activity of c(ILDV$\left.\mathrm{NH}\left(\mathrm{CH}_{2}\right)_{5} \mathrm{CO}\right)$, a novel, selective, cyclic peptide inhibitor of VLA-4mediated cell adhesion. Br J Pharmacol 1999;126:1751-1760.

31. Isobe M, Suzuki J, Yagita H, Okumura K, Yamazaki S, Nagai R, Yazaki Y, Sekiguchi M. Immunosuppression to cardiac allografts and soluble antigens by anti-vascular cellular adhesion molecule- 1 and anti-very late antigen-4 monoclonal antibodies. J Immunol 1994;153:5810-5818.

32. Sagara H, Matsuda H, Wada N, Yagita H, Fukuda T, Okumura K, Makino S, Ra C. A monoclonal antibody against very late activation antigen-4 inhibits eosinophil accumulation and late asthmatic response in a guinea pig model of asthma. Int Arch Allergy Immunol 1997;112: 287-294.

33. Ebihara N, Yokoyama T, Kimura T, Nakayasu K, Okumura K, Kanai A, Ra C. Anti VLA-4 monoclonal antibody inhibits eosinophil infiltration in allergic conjunctivitis model of guinea pig. Curr Eye Res 1999;19:20-25.

34. McDermott JR, Grencis RK, Else KJ. Leucocyte recruitment during enteric nematode infection. Immunology 2001;103:505-510.

35. Araki M, Araki K, Vassali P. Cloning and sequencing of mouse VCAM-1 cDNA. Gene 1993;126:261-264.

36. Behzad AR, Chu F, Walker DC. Fibroblasts are in a position to provide directional information to migrating neutrophils during pneumonia in rabbit lungs. Microvasc Res 1996;51:303-316.

37. Walker DC, Behzad AR, Chu F. Neutrophil migration through preexisting holes in the basal laminae of alveolar capillaries and epithelium during streptococcal pneumonia. Microvasc Res 1995;50:397-416.

38. Loosli CG, Baker RF. Acute experimental pneumococcal (type I) pneumonia in the mouse: the migration of leukocytes from the pulmonary capillaries into the alveolar spaces as revealed by the electron microscope. Trans Am Clin Climatol Assoc 1962;74:15-28.

39. Shaw JO. Leukocytes in chemotactic fragment-induced lung inflammation. Am J Pathol 1980;101:283-302.

40. Downey GP, Worthen GS, Henson PM, Hyde DM. Neutrophil sequestration and migration in localized pulmonary inflammation: capillary localization and migration across the interalveolar septum. Am Rev Respir Dis 1993;147:168-176.

41. Henderson WR Jr, Chi EY, Albert RK, Chu S-J, Lamm WJE, Rochon Y, Jonas M, Christie PE, Harlan JM. Blockade of CD49d ( $\alpha 4$ integrin) on intrapulmonary but not circulating leukocytes inhibits airway inflammation and hyperresponsiveness in a mouse model of asthma. $J$ Clin Invest 1997;100:3083-3092.

42. Horwitz BH, Mizgerd JP, Scott ML, Doerschuk CM. Mechanisms of granulocytosis in the absence of CD18. Blood 2001;97:1578-1583.

43. Li XC, Miyasaka M, Issekutz TB. Blood monocyte migration to acute 
lung inflammation involves both CD11/CD18 and very late activation antigen-4-dependent and independent pathways. J Immunol 1998;161: 6258-6264.

44. Yamamoto Y, Ikeda K, Watanabe M, Shimomura A, Suzuki H, Oshima T, Imamura Y, Ohuchi K, Tasaka T. Expression of adhesion molecules in cultured human nasal mucosal microvascular endothelial cells activated by interleukin-1 beta or tumor necrosis factor-alpha: effects of dexamethasone. Int Arch Allergy Immunol 1998;117:68-77.
45. Amberger A, Maczek C, Jurgens G, Michaelis D, Schett G, Trieb K, Eberl T, Jindal S, Xu Q, Wick G. Co-expression of ICAM-1, VCAM-1, ELAM-1 and Hsp60 in human arterial and venous endothelial cells in response to cytokines and oxidized low-density lipoproteins. Cell Stress Chaperones 1997;2:94-103.

46. Burns AB, Takei F, Doerschuk CM. Quantitation of ICAM-1 expression in mouse lung during pneumonia. J Immunol 1994;153:3189-3198. 